\title{
Wind load modeling for topology optimization of continuum structures
}

\author{
Ramzi Zakhama • Mostafa M. Abdalla • \\ Zafer Gürdal • Hichem Smaoui
}

Received: 12 February 2009 / Revised: 4 December 2009 / Accepted: 1 January 2010 / Published online: 23 January 2010

(C) The Author(s) 2010. This article is published with open access at Springerlink.com

\begin{abstract}
Topology optimization of two and three dimensional structures subject to dead and wind loading is considered. The wind loading is introduced into the formulation by using standard expressions for the drag force, and a strategy is devised so that wind pressure is ignored where there is no surface obstructing the wind. A minimum compliance design formulation is constructed subject to a volume constraint using the Solid Isotropic Material with Penalization model. The optimization problem is solved using the Method of Moving Asymptotes, modified by including a line search and by changing the formula for the update of asymptotes. To obtain black/white design, intermediate density values, which are used as design variables, are controlled by imposing an explicit constraint. Numerical examples of a windmill structure demonstrate that the proposed formulation rationally incorporates the effect of wind loading into the topology optimization problem as illustrated by void appearing in the optimal structure.
\end{abstract}

\footnotetext{
An earlier version of this paper has been presented at the Second EWEA/EAWE Special Topic Conference: The Science of Making Torque from Wind, Copenhagen, Aug. 2007 (Zakhama R, Abdalla MM, Gürdal Z, Smaoui H (2007) Wind load effect in topology optimization problems. Journal of Physics: Conference Series 75, doi:10.1088/1742-6596/75/1/012048).
}

R. Zakhama · M. M. Abdalla · Z. Gürdal Aerospace Structures, TUDelft, Kluyverweg 1, Delft 2629 HS, The Netherlands

R. Zakhama · H. Smaoui Department of Civil Engineering, National School of Engineering at Tunis, BP 37 Le Belvédère Tunis, Tunisia

R. Zakhama $(\bowtie) \cdot$ H. Smaoui

Polytechnic School of Tunisia, La Marsa 2078, Tunisia

e-mail: rzakhama@hotmail.com
Keywords Topology optimization - Continuum structures · Design dependent loads · Wind loads $\cdot$ Moving asymptotes

\section{Introduction}

Research into the optimization of the topology of continuum structures (Bendsøe and Kikuchi 1988) is well established and continues to attract the attention of researchers and software companies in various engineering fields. However, few authors take into account the effect of design dependent loads on the topology optimization problem. This class of problems is present in different engineering domains: the fluid pressure loading on structures in hydrostatic problems, snow loading in civil engineering structures, and wind and hydrodynamic loadings on off shore structures. In these problems, the location, direction, and magnitude of loading are dependent on the design of the structure and cannot be prescribed a priori.

In one of the early papers on design dependent loads Hammer and Olhoff (2000) considered the problem of topology optimization of elastic continuum structures under static pressure loading. The authors of this paper introduced a curve of iso-volumetric density to simulate the variable loading surface. In subsequent papers by $\mathrm{Du}$ and Olhoff (2004a, b), the use of a parameterized iso-volumetric density curve to represent the variable loading surface was extended. The authors used a modified isoline technique to make the process more robust for loading surface identification.

One of the early papers in this field is by Fuchs and Moses (2000) where a line of action is used to prescribe the allowable moving (transmissible) load while keeping the total magnitude constant. The load magnitude of each line of action is specified but its location along that line is left as 
part of the optimization process. Later Fuchs and Shemesh (2004) improved the earlier work (Fuchs and Moses 2000) by introducing a low modulus material in fluid regions and enforcing smooth transition between the solid and fluid regions. The new formulation resulted in improved performance in the design of hydraulically loaded structures such as dams.

Another contribution to topology optimization subject to design-dependent loading is attributed to Chen and Kikuchi (2001). In this work, the authors proposed an approach to simulate the design-dependent loads using fictitious thermal loads. The topology optimization problem is transformed from a two-phase to a three-phase material distribution problem within the design domain in which the solid, void, and hydrostatic fluid phases are optimally distributed. More recently, Sigmund and Clausen (2007) have suggested a new way to solve pressure load problems in topology optimization by using a mixed displacement-pressure formulation for the underlying finite element problem.

The main ideas described above are not all applicable to the wind loading problem addressed in this paper. For example, wind loading cannot be represented using the method of transmissible loads, especially in three dimensions, since no wind loading is applied when there is no surface obstructing the wind. Although the iso-volumetric surface method can be applied, it is interesting to develop a direct density based method that does not involve explicit construction of the loading surfaces.

In this paper, wind loading is introduced into the topology optimization formulation for two and three dimensional continuum structures by using the standard formula for drag forces as initially proposed in Zakhama et al. (2007) for two dimensional problems. An optimization problem is constructed as a compliance minimization problem and is solved by a modified Method of Moving Asymptotes (MMA) (Svanberg 1987). ${ }^{1}$ The lower asymptote is modified according to the continuous optimality criteria interpreted as local Kuhn-Tucker conditions (Abdalla and Gürdal 2002). Due to the non-monotonous convergence of compliance, the coefficients of the approximation of the objective function suggested by Svanberg (1987) are also modified. A line search (Zillober 1993) is applied to ensure decrease in the objective function and to satisfy the constraints. Finally, an explicit function is introduced as a constraint to obtain a black/white design.

The rest of this paper is organized as follows. Formulation of the standard topology optimization problem is given in Section 2. Topology design with wind loading is described in Section 3. An explicit constraint is defined

\footnotetext{
${ }^{1}$ Prof. Svanberg also provided us with the computer code used in this research.
}

in Section 4 to lead the topology to a black/white design. Numerical examples are presented in Section 5 followed by conclusions in Section 6.

\section{Standard topology optimization formulation}

The standard objective of a topology optimization problem is to find the optimal material distribution within a given domain. In this paper, the topology optimization problem is posed following the commonly used minimum compliance formulation with a material volume constraint

$$
\begin{array}{r}
\min _{\rho} W\left(\mathbf{u}^{*}\right)=\mathbf{F} \cdot \mathbf{u}^{*} \\
\text { subject to } \\
V=\int_{\Omega} \rho d \Omega \leq V^{*} \\
0 \leq \underline{\rho} \leq \rho \leq 1,
\end{array}
$$

where $W$ is the compliance of the structure which can be written either as the work done by the external forces or as twice the total elastic energy at equilibrium. The vector $\mathbf{F}$ denotes the external forces, and $\mathbf{u}^{*}$ is the displacement field of the domain $\Omega$ at equilibrium. The local density distribution of material $\rho$ of the discretized model is chosen as a design variable, and $\rho$ is a small number set as a lower bound on $\rho$ to avoid numerical instability. The bound $V^{*}$ is the allowable limit on the material volume $V$ of the structure.

The well known SIMP (Solid Isotropic Material with Penalization) approach (Rozvany et al. 1992) was selected to achieve black/white topology designs. The local material stiffness $E$ is defined as

$E=\bar{\rho}^{p} E_{0}$,

where $E_{0}$ is the base elasticity modulus, and $p$ is a penalization parameter $(p \geq 3)$. In the present formulation the local density measures $\rho_{i}$ of the discretized structure are associated with the nodes of the finite element mesh. The element densities $\bar{\rho}$ are obtained by an average compliance interpolation (Abdalla and Gürdal 2002) for the $m$ nodes surrounding the element and given by

$\frac{1}{\bar{\rho}^{p}}=\frac{1}{m} \sum_{i=1}^{m} \frac{1}{\rho_{i}^{p}}$.

Using this scheme checkerboard patterns are suppressed automatically during the optimization process. 


\section{Topology design with wind loading}

This section begins with an explanation of how the wind loading is introduced into the topology optimization problem. A sensitivity analysis is then carried out. Finally, the optimization problem is solved using the modified Method of Moving Asymptotes (MMA) (Svanberg 1987) and the line search method.

\subsection{Wind loading function}

Wind typically blows in many directions at varying speeds. Ideally multiple load cases need to be considered in the topology optimization process to account for the consequent variabilities in wind forces acting on a structure. However, in this paper only one load case is considered for simplicity; with uni-directional incoming wind blowing from left to right (see Fig. 1a). This assumption remains valid for any incoming wind directions. Wind loading is included in the formulation using a direct density based method that does not involve explicit construction of the loading surfaces. The wind load is defined at each node of the discretized domain by introducing a loading function $g$ using the standard formula for drag forces

$$
F_{\text {Wind }_{C}}=C_{D} Q_{\infty} A g,
$$

where $F_{\text {Wind }_{C}}$ is the wind load value at the node $C, Q_{\infty}$ is the incoming wind dynamic pressure, $C_{D}$ is the drag coefficient, and $A$ is the area of the surface side facing the wind in $Y Z$ directions. Computation of the area $A$ depends on the node position of $C$, i.e. whether the node is at a corner, a side, or the middle of the discretized domain. For example for a middle node, in the 2D case, A is equal to $a$ times the thickness of the structure and in the 3D case it is equal to $a^{2}$ where $a$ is the distance between the nodes of a uniform mesh. The factor $g$ is a peak function interpolation (Yin and Ananthasuresh 2001) given by

$$
g=e^{-\frac{\left[1-\left(\rho_{C}-\rho_{N}\right)\right]^{2}}{2 \sigma^{2}}},
$$

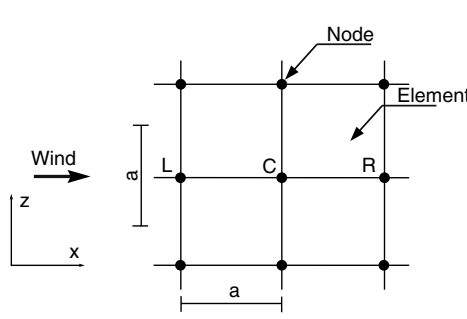

(a) Wind direction.

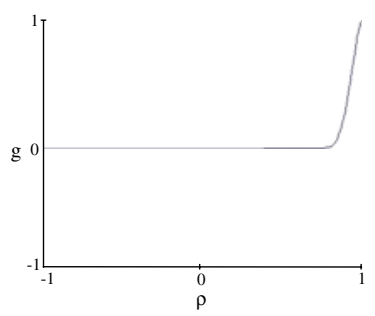

(b) Peak function.
Fig. 1 Wind direction and peak function where $\rho_{C}$ is the density of the node $C$ and $\rho_{N}$ is the density of the neighboring node in the direction opposite to wind direction $\left(\rho_{N}=\rho_{L}\right.$ when the incoming wind is from left to right), and $\sigma$ is a standard deviation parameter.

Yin and Ananthasuresh (2001) proposed to use the peak function to include multiple materials in the design without increasing the design variables. By adjusting the parameters in the model, multiple peaks can be created, so that, the final solution can be made to posses pure materials by avoiding intermediate values. In this paper, the force $F_{W i n d}$ is applied at every node of the domain and the peak function $g$ is used to steer its value towards to 0/1 (see Fig. 1b), which ensures that the wind loading is applied only at nodes where a large change in density occurs, indicating a structural surface, and that the loading is unidirectional. In other words, the load is applied only when the node $C$ is in a solid region and the node $N$ is in void. In the present formulation, we are interested in truss like structures. Thus, we make the assumption that no wind shadowing is present.

\subsection{Sensitivity analysis}

The sensitivity of the compliance with respect to the design variables, which are the node densities in this case, is obtained from

$\frac{d W}{d \rho}=\frac{d \mathbf{F}^{T}}{d \rho} \mathbf{u}+\mathbf{F}^{T} \frac{d \mathbf{u}}{d \rho}$.

The sensitivity of the displacement in the above equation can be obtained by differentiating the equilibrium equation with respect to the node densities and is expressed as

$\frac{d \mathbf{u}}{d \rho}=\mathbf{K}^{-1}\left(\frac{d \mathbf{F}_{\text {Wind }}}{d \rho}-\frac{d \mathbf{K}}{d \rho} \mathbf{u}\right)$,

where $\mathbf{K}$ is the global stiffness matrix and $\mathbf{F}_{\text {Wind }}$ is the wind load vector. Thus, the sensitivity of the compliance is given by

$$
\frac{d W}{d \rho}=2 \mathbf{u}^{T} \frac{d \mathbf{F}_{\text {Wind }}}{d \rho}-\mathbf{u}^{T} \frac{d \mathbf{K}}{d \rho} \mathbf{u}
$$

As can be observed from (8), the sensitivity of the compliance is decomposed into two terms; the first term is due to the dependence of external forces on the design variables, and the second one is due to the dependence of the stiffness on the same variables. We denote these two terms by $\psi$ and $\phi$, respectively. Furthermore, external forces applied to a given structure can be decomposed into two parts, which are the static loads and the incoming wind forces. Dead loads are assumed to be independent of the structural 
configuration. Thus, the expression of $\psi$ for each node $C$ depends only on wind loading and simplifies to

$$
\begin{aligned}
\psi_{C}=\frac{C_{D} Q_{\infty} A}{\sigma^{2}}\{[ & {\left[1-\left(\rho_{C}-\rho_{L}\right)\right] e^{\left.-\frac{\left[1-\left(\rho_{C}-\rho_{L}\right)\right]^{2}}{2 \sigma^{2}}\right] u_{C}} } \\
& \left.-\left[\left[1-\left(\rho_{R}-\rho_{C}\right)\right] e^{-\frac{\left[1-\left(\rho_{R}-\rho_{C}\right)\right]^{2}}{2 \sigma^{2}}}\right] u_{R}\right\},
\end{aligned}
$$

where the subscripts $L$ and $R$ refer to the nodal points to the left and to the right of node $C$, respectively, and the wind direction is assumed to be from left to right. Using the same notation, $u_{C}$ and $u_{R}$ are the horizontal displacement at node $C$ and its right neighbor, respectively.

\subsection{Optimization}

The optimization problem is solved by the Method of Moving Asymptotes (MMA) (Svanberg 1987). According to Svanberg (1987), the compliance is approximated as

$W^{(k)}(\rho)=r_{0}^{(k)}+\sum_{j=1}^{n}\left(\frac{p_{0 j}^{(k)}}{U_{j}^{(k)}-\rho_{j}}+\frac{q_{0 j}^{(k)}}{\rho_{j}-L_{j}^{(k)}}\right)$,

where $\mathrm{k}$ is the MMA iteration index, $n$ is the total number of nodes of the discretized domain, $p_{0 j}^{(k)}$ and $q_{0 j}^{(k)}$ are determined by a first-order approximation of the first KuhnTucker condition, and $L_{j}^{(k)}$ and $U_{j}^{(k)}$ are the lower and upper asymptotes, respectively.

It is observed that, in some cases, the optimization process tends to oscillate when the MMA solver tries to update the asymptotes $L_{j}^{(k)}$ and $U_{j}^{(k)}$. The convergence is stabilized by modifying the lower asymptote according to the continuous optimality criteria interpreted as local Kuhn-Tucker conditions (Abdalla and Gürdal 2002). The update of the upper asymptote is kept the same as suggested by Svanberg (1987). Using the continuous optimality criteria interpreted as local Kuhn-Tucker conditions the objective function is approximated as

$W^{(k)}=\sum_{j=1}^{n} \frac{\phi_{j}^{(k)}}{\left(\rho_{j}^{(k)}\right)^{p}}$.

This approximation can be convexified by linearizing the stiffness coefficient $\rho_{j}^{p}$ around the most recent design point to obtain

$W^{(k)}=\sum_{j=1}^{n} \frac{q_{0 j}^{(k)}}{\rho_{j}^{(k)}-L_{j}}$,

where $L_{j}=\left(1-\frac{1}{p}\right) \rho_{j}^{(k-1)}$ is the modified formula for the lower asymptote.
With the modifications discussed above the MMA solver does not always converge monotonously and in some cases it diverges, especially for strong wind loadings. To improve its performance, the calculation of the coefficients $p_{0 j}^{(k)}$ and $q_{0 j}^{(k)}$ defined in Svanberg (1987) is modified by considering the sensitivity contributions from the $\phi$ and $\psi$ terms independently,

$$
\begin{aligned}
p_{0 j}^{(k)}= & \begin{cases}\left(U_{j}^{(k)}-\rho_{j}^{(k)}\right)^{2} \psi_{j}, & \psi_{j}>0 \\
0, & \psi_{j} \leq 0\end{cases} \\
q_{0 j}^{(k)}= & \left(\rho_{j}^{(k)}-L_{j}^{(k)}\right)^{2} \phi_{j} \\
& + \begin{cases}0, & \psi_{j} \geq 0 \\
-\left(\rho_{j}^{(k)}-L_{j}^{(k)}\right)^{2} \psi_{j}, & \psi_{j}<0 .\end{cases}
\end{aligned}
$$

Moreover, it is also observed that the objective function does not always decrease after an MMA step and that the constraints are violated in some cases. In an earlier paper, Zillober (1993) demonstrated that, by adding a line search in the optimization process the behavior of the MMA method can be stabilized. Therefore, a line search is used in the present work to ensure satisfaction of the constraints and decrease in the objective value. Let the solution produced by MMA after the $k^{\text {th }}$ iteration be denoted by $\rho^{*}$. A solution is sought in the form

$\rho^{(k+1)}=\left(\rho^{*}-\rho^{(k)}\right) \alpha+\rho^{(k)}$,

where $\alpha \in[0,1]$ is a parameter that needs to be determined such that the constraints be satisfied and the objective function be decreased. The value of $\alpha$ is found by solving a one-dimensional approximate optimization problem constructed from the original problem by using approximate cubic polynomial approximations for the objective and the constraints as functions of the independent variable $\alpha$.

\section{Explicit constraint}

Preliminary results for a numerical example illustrating an off-shore wind turbine support structure exhibit a grey design as the optimal topology (see Fig. 2a) as opposed to a black/white one as expected. The explanation for this is related to the distribution of the wind loads over the nodes as shown in Fig. 2b. Each column in the figure is a histogram of the magnitude of the loads on nodes along a specific vertical line. Note that the wind loads are distributed throughout the domain, but have significant magnitude only for a few nodes at the bottom of the structure; but, the distribution of the 
Fig. 2 Off-shore wind turbine problem without explicit constraint

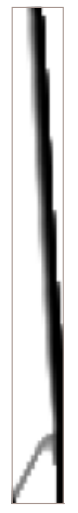

(a) Topology with wind.

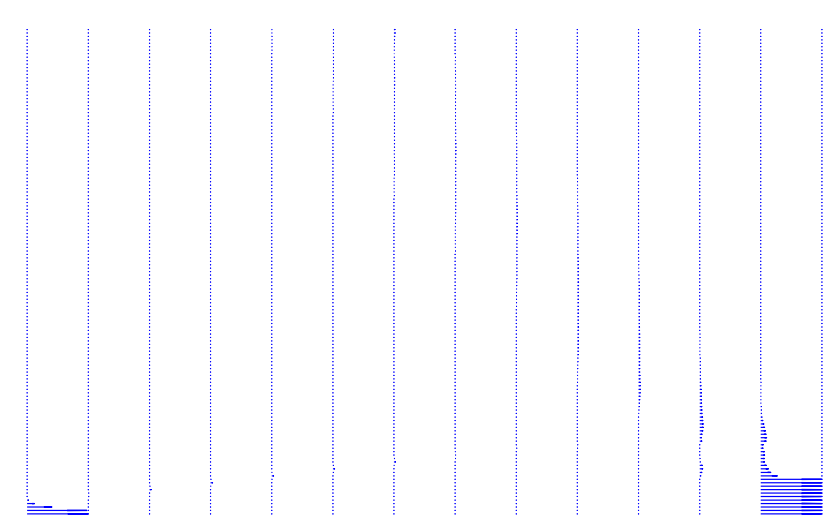

(b) Wind load distribution for each node of the discretized domain. wind loads must be uniform on a surface facing the wind, which is not the case. To generate a black/white designs, an explicit constraint (Borrvall and Petersson 2001) was added to the formulation of problem (1) as a means to control the intermediate densities. The explicit constraint is defined as

$h(\rho)=\int_{\Omega}(1-\rho)(\rho-\underline{\rho}) d \Omega \leq \epsilon_{p}$.

In its numerical implementation the explicit constraint (16) is enforced gradually as follows. Initially, it is ignored, which results in a grey design. The value of the constraint function for this design is denoted by $\epsilon_{p}^{(0)}$. Next, a sequence of problems $P_{k}$ are solved, each subject to the additional constraint (16) where the right hand side $\epsilon_{p}$ is the $k^{\text {th }}$ term updated according to the formula

$\epsilon_{p}^{(k)}=\epsilon_{p}^{(k-1)}(1-\beta)$.

where the reduction $\beta$ is defined by the user.

Fig. 3 Design domain of an associated decreasing sequence initialized at $\epsilon_{p}^{(0)}$ and
The process continues until the solver fails to converge for the updated value of $\epsilon_{p}$.

\section{Numerical examples}

To evaluate the influence of wind loading on the optimum topology, the numerical example of an off-shore wind turbine support structure is considered. The design domain is a parallelepiped volume as shown in Fig. 3 which reduces to a rectangular domain in the $2 \mathrm{D}$ case. The hub and rotor weight $P_{W R}$, and the rotor thrust $F_{W}$ are considered as dead loads and they are applied at the center of the top side of the domain with values of $2.55 \mathrm{MN}$ and $0.125 \mathrm{MN}$, respecwith dimensions of $120 \mathrm{~m}$ in height, $12 \mathrm{~m}$ in length and $12 \mathrm{~m}$ in width. The structure is clamped at the bottom. The incoming wind is assumed to flow from left to right with a wind speed of $25 \mathrm{~m} / \mathrm{s}$. The problem is studied in both two and three dimensional cases with the assumption of linear small deformations. The material used is steel with Young's

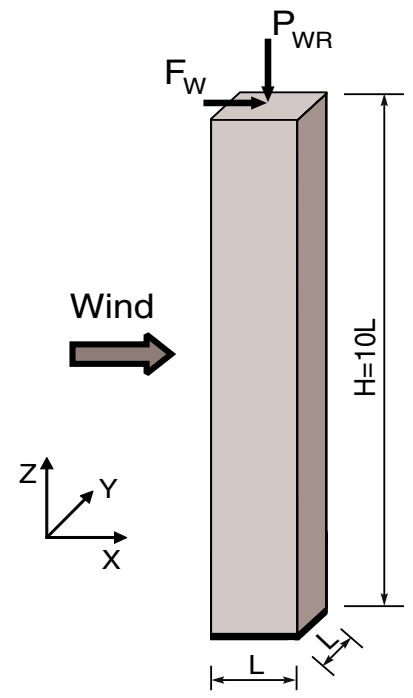

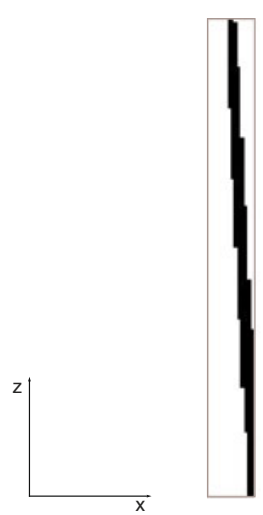

(a) Optimum topology without wind.

(b) Optimum Topology with wind.
Fig. 4 Optimum 2D topology of an off-shore wind turbine tively. The spatial design domain considered is box shaped 
Fig. 5 Evolution of the wind turbine support topology

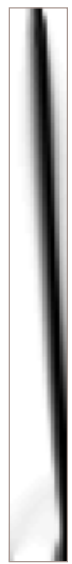

(a) after 20 iterations.

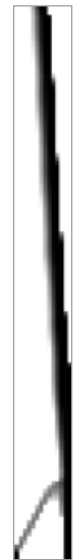

(b) after 100 iterations.
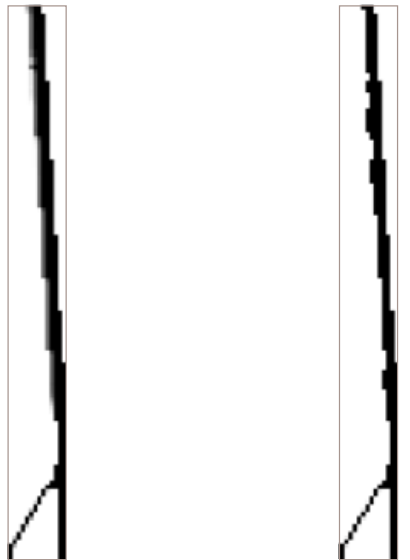

(c) after 300 iterations.

(d) after convergence.

modulus $E=200,000 \mathrm{MPa}$, and Poisson's ratio $v=0.3$. The penalization parameter is set to 3 , the volume fraction is set to be 0.3 , and a lower bound $\rho=10^{-3}$ is adopted for the density. The value of $2 \sigma^{2}$ for the peak function is fixed at 0.1 and the value of $\beta$ to 0.05 .

\subsection{Two dimensional example}

A 2D problem is first considered to investigate the topology optimization of the wind turbine support structure. The results of this problem were generated with a discretized grid of $15 \times 141$ nodes in the $L$ and $H$ directions, respectively. The final designs are represented in Fig. $4 \mathrm{a}$ and $\mathrm{b}$ for the cases with and without wind loading, respectively, while maintaining the hub and rotor weight $P_{W R}$, and the rotor thrust $F_{W}$ for the both cases. It can be observed that the topology without wind is a leaning tower that supports only the dead loads. On the other hand, the topology subject to wind loads has a tie forming at the root. Moreover, it can be seen from Fig. 4a, b that the tower is a narrow cantilever as opposed to having a thick root. This is due to the magnitude difference between the two forces; $P_{W R}$ is about 20 times larger than $F_{W}$. The compliances computed with dead and wind loads for both optimum structures are $0.0229 \mathrm{MNm}$ for the topology without wind loading and $0.0139 \mathrm{MNm}$ for the topology with wind loading, respectively. Thus, including wind loading in the design formulation leads to $40 \%$ increase in structural stiffness for the same material volume. If the force $F_{W}$ were to be applied at the front end of the top surface, the tower would have been leaning more and possibly produce a better optimum.

Figure 5 shows the evolution of the wind turbine support topology. The algorithm converges to a black/white topology in about 884 analysis iterations. It can be observed that the topology starts from a grey column design and ends up with a black/white design with apparition of a tie forming at the root that supports the loads coming from the wind.

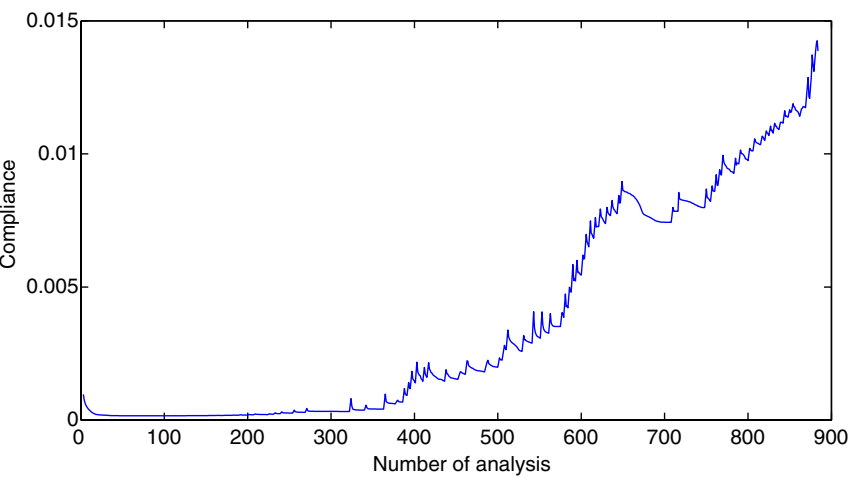

(b) Convergence history. domain.

Fig. 6 Wind distribution and convergence history 


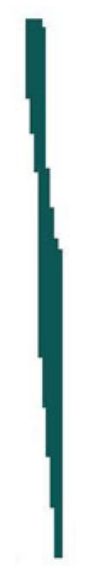

(a) XZ view without wind.

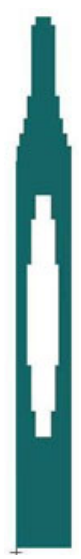

(c) YZ view without wind.

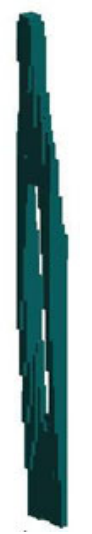

(e) 3D view without wind.

(f) 3D view with wind.

Fig. 7 3D topology of the wind turbine support

Figure 6a shows the distribution of wind loads for the design obtained considering wind loading. It can be observed that the wind loads are uniformly distributed throughout the design domain. In some cases, especially for strong wind (Zakhama et al. 2007), the wind loads may be not uniformly distributed in some parts of the domain. This is due mainly to the way the coefficients $p_{0 j}^{(k)}$ and $q_{0 j}^{(k)}$ of the compliance approximation are updated and the parameter $\epsilon_{p}$ of the explicit constraint is reduced. Further investigation is needed to determine an appropriate update scheme for these coefficients. Figure $6 \mathrm{~b}$ shows the convergence history. The convergence is reasonably smooth with jumps in compliance corresponding to tightened tolerance on grey density. Although the introduction of the explicit constraint leads to an increased number of iterations to convergence, the obtained solution is physically more meaningful.

\subsection{Three dimensional example}

The off-shore wind turbine domain (see Fig. 3 ) is now discretized with $15 \times 15 \times 141$ nodes. Figure 7 shows the different views of the optimal topology of wind turbine support without and with wind loads. To compare the compliances of the two solutions, the designs obtained with and without wind loads are postprocessed to force exact black/white topologies. The compliance without considering wind loads is $0.0239 \mathrm{MNm}$ and the compliance considering wind loads is $0.0151 \mathrm{MNm}$, which corresponds to $37 \%$ difference between the two solutions.

The topologies obtained for the 3D case are seen to be very similar to the topologies obtained for the $2 \mathrm{D}$ case in $\mathrm{XZ}$ plane (see Figs. 4 and 7a, b). The topology of the wind turbine support considering wind loading is supported by two frames at the bottom of the structure. Figure $7 \mathrm{~d}$ shows that, by including wind loading in the formulation, the topology allowed a larger hole facing the wind loads than the topology without wind. In the literature, most techniques for topology optimization with design dependent loads do not allow holes facing a surface loads.

\section{Conclusions}

A formulation for the inclusion of wind loading in the minimum compliance topology optimization problem has been proposed. The method does not require the explicit construction of loading surfaces. The MMA method has been modified and a line search has been added to guarantee the convergence process of the topology optimization problem. An explicit constraint has been added into the topology optimization formulation to control the intermediate density values and to ensure black/white topology. Numerical examples demonstrate the effect of the wind loads on the optimized topologies. Taking the wind loads into account in the formulation gives stiffer designs with the same volume and can allow holes facing the wind loads. Thus, the implementation of this method in the preliminary design phase can lead to significant reduction in structural weight 
which is reflected on the total cost of off-shore wind farms especially given the rising steel prices.

Acknowledgments This research was supported by the National Science Foundation, Grant NSF OISE-0353186. We wish many thanks to Prof. Svanberg for providing us with the computer code used in this research.

Open Access This article is distributed under the terms of the Creative Commons Attribution Noncommercial License which permits any noncommercial use, distribution, and reproduction in any medium, provided the original author(s) and source are credited.

\section{References}

Abdalla MM, Gürdal Z (2002) Structural design using optimality based cellular automata. In: 43th AIAA/ASME/AHS/ASC structures, structural dynamics and material conference, Denver, CO

Bendsøe MP, Kikuchi N (1988) Generating optimal topologies in structural design using homogenization method. Comput Methods Appl Mech Eng 71:197-224

Borrvall T, Petersson J (2001) Topology optimization using regularized intermediate density control. Comput Methods Appl Mech Eng 190:4911-4928

Chen BC, Kikuchi N (2001) Topology optimization with designdependent loads. Finite Elem Anal Des 37:57-70

Du J, Olhoff N (2004a) Topological optimization of continuum structures with design-dependent surface loading — part I: new com- putational approach for 2D problems. Struct Multidisc Optim $27: 151-165$

Du J, Olhoff N (2004b) Topological optimization of continuum structures with design-dependent surface loading — part II: algorithm and examples for 3D problems. Struct Multidisc Optim 27: $166-177$

Fuchs MB, Moses E (2000) Optimal structural topologies with transmissible loads. Struct Multidisc Optim 19:263-273

Fuchs MB, Shemesh NNY (2004) Density-based topological design of structures subjected to water pressure using a parametric loading surface. Struct Multidisc Optim 28:11-19

Hammer VB, Olhoff N (2000) Topology optimization of continuum structures subjected to pressure loading. Struct Multidisc Optim 19:85-92

Rozvany GIN, Zhou M, Birker T (1992) Generalized shape optimization without homogenization. Struct Multidisc Optim 4: $250-252$

Sigmund O, Clausen PM (2007) Topology optimization using a mixed formulation: an alternative way to solve pressure load problems. Comput Methods Appl Mech Eng 196:1874-1889

Svanberg K (1987) The method of moving asymptotes-a new method for structural optimization. Int J Numer Methods Eng 24: 359-373

Yin L, Ananthasuresh GK (2001) Topology optimization of compliant mechanisms with multiple materials using a peak function material interpolation scheme. Struct Multidisc Optim 23: $49-62$

Zakhama R, Abdalla MM, Gürdal Z, Smaoui H (2007) Wind load effect in topology optimization problems. J Phys Conf Ser 75. doi:10.1088/1742-6596/75/1/012048

Zillober C (1993) A globally convergent version of the method of moving asymptotes. Struct Optim 6:166-174 